\title{
A Vector Network Analyzer Based on Pulse Generators
}

\author{
B. Schulte, M. Gerding, and B. Schiek \\ RF \& Microwave Engineering Institute, Ruhr-University of Bochum, Universitätsstraße 150, 44780 Bochum, Germany
}

\begin{abstract}
A fast four channel network analyzer is introduced to measure $S$-parameters in a frequency range from $10 \mathrm{MHz}$ to $3 \mathrm{GHz}$. The signal generation for this kind of analyzer is based on pulse generators, which are realized with bipolar transistors. The output signal of the transistor is differentiated and two short pulses, a slow and a fast one, with opposite polarities are generated. The slow pulse is suppressed with a clipping network. Thus the generation of very short electrical pulses with a duration of about $100 \mathrm{ps}$ is possible. The structure of the following network analyzer is similar to the structure of a conventional four channel network analyzer.

All four pulses, which contain the high frequency information of the device under test, are evaluated after the digitalization of intermediate frequencies. These intermediate frequencies are generated with sampling mixers. The recorded data is evaluated with a special analysis technique, which is based on a Fourier transformation.

The calibration techniques used are the same as for conventional four channel network analyzers, no new calibration techniques need to be developed.
\end{abstract}

\section{Introduction}

Special applications of metrology often require fast measurements of complex-valued scattering parameters. One example of such an application is a mass production in the industry. A measurement system for these applications can be based on pulse generators. The advantages of these generators are on the one hand the short measuring time and on the other hand the cost-effective realization of the signal generators.

In conventional systems every frequency point is evaluated separately. Due to the fact that no discrete frequency points are available here, the pulse response signals are recorded completely in the time domain. Therefore a special analysis technique is necessary. This special analysis technique is based on a Fourier transformation of the pulse signal. For high precision e.g. in laboratories the fast network analyzer should be able to perform calibrated measurements.

Correspondence to: B. Schulte

(bernd.schulte@ruhr-uni-bochum.de)

\section{Structure of the Pulse Generator}

The pulse generator, which is used for the signal generation, is shown in Fig. 1 (Gerding, 2003).

The generator consists of four sections: a driver circuit, a slope enhancement circuit, a differentiation network and a clipping network. The driver circuit uses an edge-triggered input stage in order to provide a trigger signal to the slope enhancement circuit. The slope enhancement circuit creates a steeper pulse which is nearly independent of the waveform, the duty-cycle and the amplitude of the input signal of the driver circuit. With the leading edge of the trigger signal, the bipolar transistor of the slope enhancement circuit is driven into a saturation mode. The trailing edge of the trigger signal causes a depletion of the stored charge in the base-collector junction, followed by a sudden increase of the base-collector impedance. Then also the voltage across the collector-emitter junction changes abruptly. This leads to a short rise time of the trailing edge. In the next section of the pulse generator the differentiation of the rectangular output signal causes a waveform, which contains two pulses with different pulse polarities and differently wide pulses. This is due to the fact that not only the fast trailing edge of the signal is differentiated, but also the slower leading edge. The clipping network contains a passive diode, which has such a polarity that it suppresses the pulse with low amplitude and wide pulse width related to the leading edge. The resulting output signal of the pulse generator now only contains the single-sided short pulse of high amplitude related to the trailing edge. Such a measured pulse signal is shown in Fig. 2.

It has an amplitude of $2.2 \mathrm{~V}$ and a $3 \mathrm{~dB}$ pulse width of $\tau=145 \mathrm{ps}$. Thus the corresponding spectral bandwidth is $B \sim \frac{1}{\tau} \approx 4.5 \mathrm{GHz}$.

\section{Structure of the Network Analyzer}

The structure of the time domain network analyzer based on pulse generators is similar to the structure of a conventional frequency domain four channel network analyzer. A block diagram of this structure is shown in Fig. 3.

The experimental network analyzer contains several pulse generators (PG). $\mathrm{PG}_{5}$ and $\mathrm{PG}_{6}$ are used to generate the measurement signals $V_{s}$. $\mathrm{PG}_{1}$ to $\mathrm{PG}_{4}$ generate the signals $V_{p}$, 


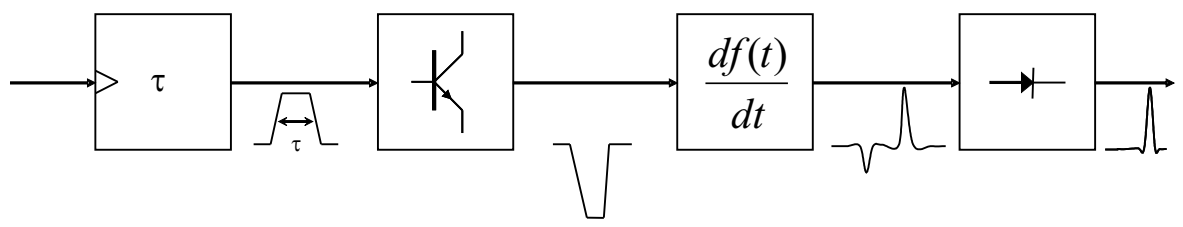

Fig. 1. Structure of a transistor based pulse generator.

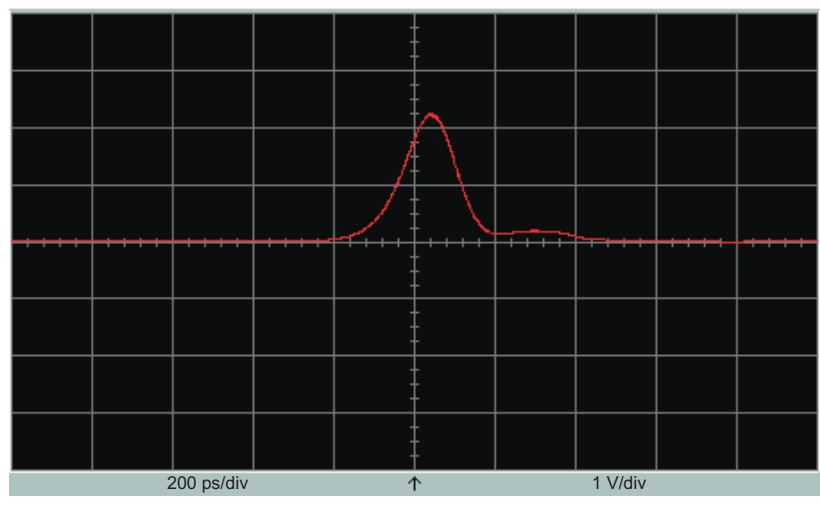

Fig. 2. Measured output signal of the pulse generator.

which serve as the local oscillator signals (L.O.) and drive the sampling mixers into a state of low conduction for a short time. The two trigger signals $V_{t 1}$ and $V_{t 2}$ of the pulse generators have a pulse repetition rate of approximately $2 \mathrm{MHz}$ with a fixed frequency difference of $74.67 \mathrm{~Hz}$. In order to keep this small frequency difference stable, the pulse repetition frequencies are stabilized with a PLL system.

The first trigger signal $V_{t 1}$ is led via a switch to either the pulse generator $\mathrm{PG}_{5}$ or the pulse generator $\mathrm{PG}_{6}$, which generate the measurement signals $V_{s}^{\mathrm{I}}$ and $V_{s}^{\mathrm{II}}$. By this means a very good isolation of the two measurement signals is achieved. The signal $V_{s}$ is led via the coupler to the device under test (DUT) and to the sampling mixers. At the sampling mixers the signal is sampled by the pulse signals $V_{p 1}$ to $V_{p 4}$. The pulse generators $\mathrm{PG}_{1}$ to $\mathrm{PG}_{4}$ of the sampling mixers are controlled by the trigger signal $V_{t 2}$. Since the pulse width of the effective sampling pulses and the measuring pulses are similar, the frequency bandwidth $B$ is reduced to an effective bandwidth of $B_{\text {eff }} \approx \frac{B}{\sqrt{2}}=3.3 \mathrm{GHz}$. The intermediate frequency $f_{i f}$ is filtered behind the amplifier by a low pass filter and digitized with an analog digital converter.

The measurement period of the time domain network analyzer is $4 \mathrm{~ms}$ with 5000 recorded points and a sampling rate of $2.5 \mathrm{MHz}$. This time is caused by two switch settings, which are necessary for one measurement. Each switch setting takes $2 \mathrm{~ms}$.

The structure of the network analyzer based on pulse generators is similar to the structure of a conventional four channel network analyzer. Thus all known calibration techniques (Schiek, 1999) can be employed for the experimental pulse network analyzer.

\section{Analysis Technique}

The data processing of the network analyzer based on pulse generators differs from the data processing of a conventional network analyzer. For the experimental network analyzer, pulse signals are recorded in the time domain in contrast to the conventional one, where every single frequency point is evaluated separately. Therefore a special analysis technique is necessary.

To explain this special technique one measurement channel is shown in Fig. 4 as a mathematical model.

For the data processing it is necessary to obtain the frequency response $F(j \omega)$ of the device under test. For this purpose the pulse signal $x_{S}(t)$ is led to the device under test. From there the signal $y(t)$ is transmitted to the sampling mixer with the frequency response $H(j \omega)$. The output signal of the mixer $z(t)$ is digitized. After a Fourier transformation of $z(t)$ the following expression is obtained:

$z(t) \circ \longrightarrow Z(j \omega)=X_{s}(j \omega) F(j \omega) H(j \omega)$

In order to receive the frequency response $F(j \omega)$, the quantities $X_{s}(j \omega)$ and $H(j \omega)$ must be eliminated. This can be done by building ratios of different Fourier transformations, because $X_{S}(j \omega)$ as well as $H(j \omega)$ after a calibration are time-invariant and will cancel. These ratios between different Fourier transformations of intermediate frequencies are needed anyway to calculate the $S$-parameters. An example for the transmission coefficient $S_{21}$ is the ratio of the wave, which is transmitted through the device under test, and the wave which is incident to the device under test.

$\mathrm{S}_{21}=\frac{Z_{3}(j \omega)}{Z_{1}(j \omega)}=\frac{X_{s}(j \omega) F_{3}(j \omega) H(j \omega)}{X_{S}(j \omega) F_{1}(j \omega) H(j \omega)}=\frac{F_{3}(j \omega)}{F_{1}(j \omega)}$

Therefore this evaluation method is a convenient method in order to calculate $S$-parameters.

\section{Measurements}

A comparison between a conventional network analyzer (a ZVR from Rohde \& Schwarz) and the network analyzer based on pulse generators was performed in order to demonstrate the functionality of this network analyzer. A low pass filter with a cut-off frequency of $300 \mathrm{MHz}$ was chosen as a 


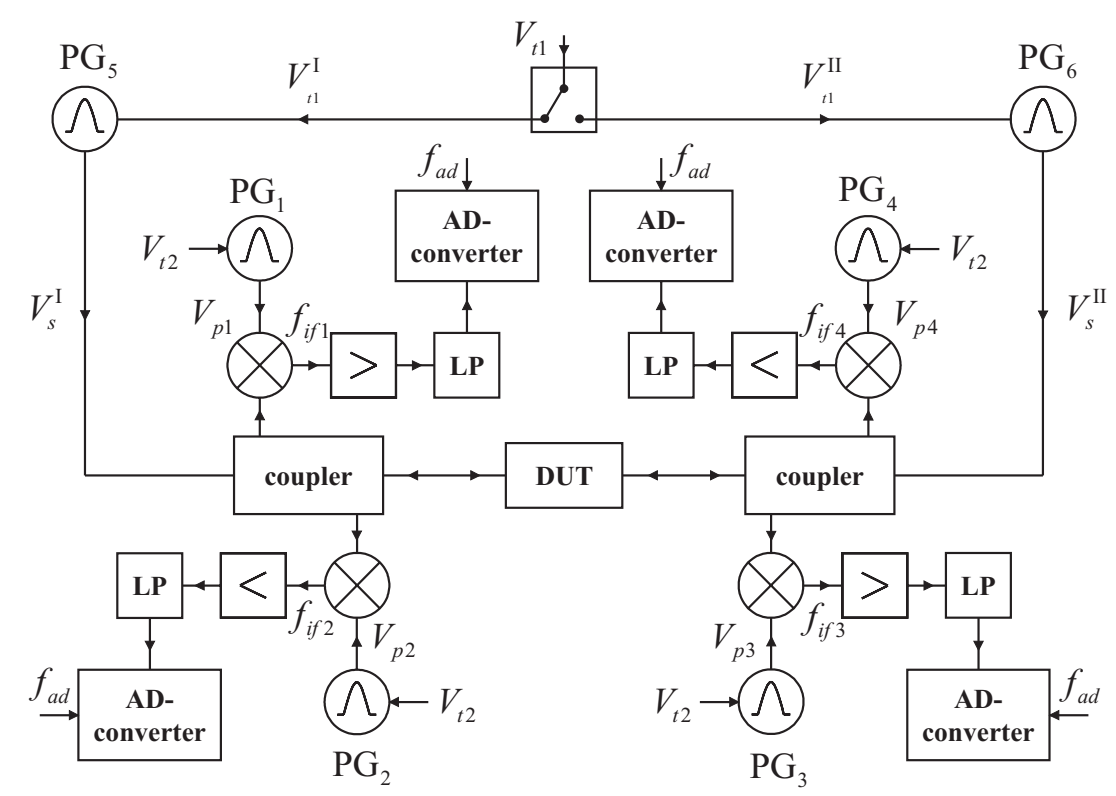

Fig. 3. Block diagram of the network analyzer.

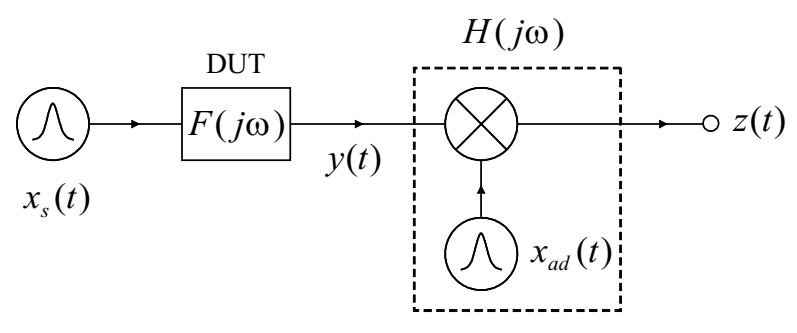

Fig. 4. Model of a measuring port.

device under test. For this measurement the conventional network analyzer has a record time of $200 \mathrm{~ms}$ with 501 points. In contrast the network analyzer based on pulse generators has a record time of $4 \mathrm{~ms}$ with 1700 points. Both network analyzers were calibrated with coaxial standards by use of the calibration method TOM (Through, Open, Match). In the figures 5 and 6 the magnitude and the phase of the transmission coefficient $S_{21}$ are displayed for a frequency range from $10 \mathrm{MHz}$ to $3 \mathrm{GHz}$.

The results of the measurements demonstrate the functionality up to $40 \mathrm{~dB}$ dynamic range of the experimental fast network analyzer. The values for the magnitude as well as for the phase are nearly identical to the values of the conventional network analyzer. A difference appears in the dynamic range of both network analyzers. The dynamic range of the experimental network analyzer, so far, is only about $40 \mathrm{~dB}$. This is much lower than the dynamic range of the conventional analyzer, which is about $90 \mathrm{~dB}$. The difference is due to the sampling principle and in addition to a cross-talk signal in the circuit of the experimental analyzer. An improvement of the dynamic range to about $60 \mathrm{~dB}$ seems to be possible as expected from simulations.

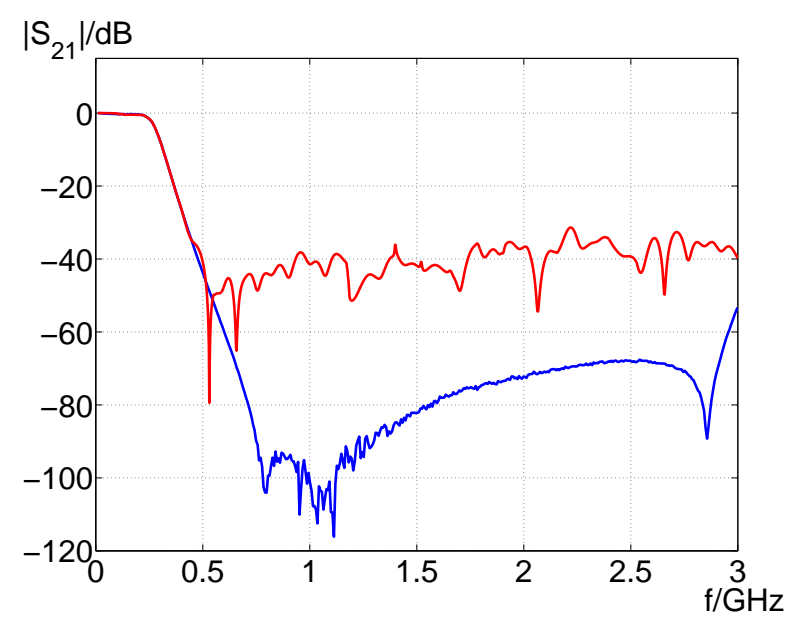

Fig. 5. Magnitude of $S_{21}$ of a $300 \mathrm{MHz}$ low pass filter, - network analyzer based on pulse generators, - commercial network analyzer (ZVR).

In Figs. 7 and 8 the magnitude and the phase of the reflection coefficient $S_{11}$ are shown, respectively. Here the measurement values for the magnitude as well as for the phase are nearly identical to the values of the conventional network analyzer. The small differences at higher frequencies result from the lower dynamic range as shown in the measurements before.

Thus the functionality of the fast network analyzer as regards the structure of the analyzer, the special analysis technique and the possibility to apply conventional calibration techniques is demonstrated. 


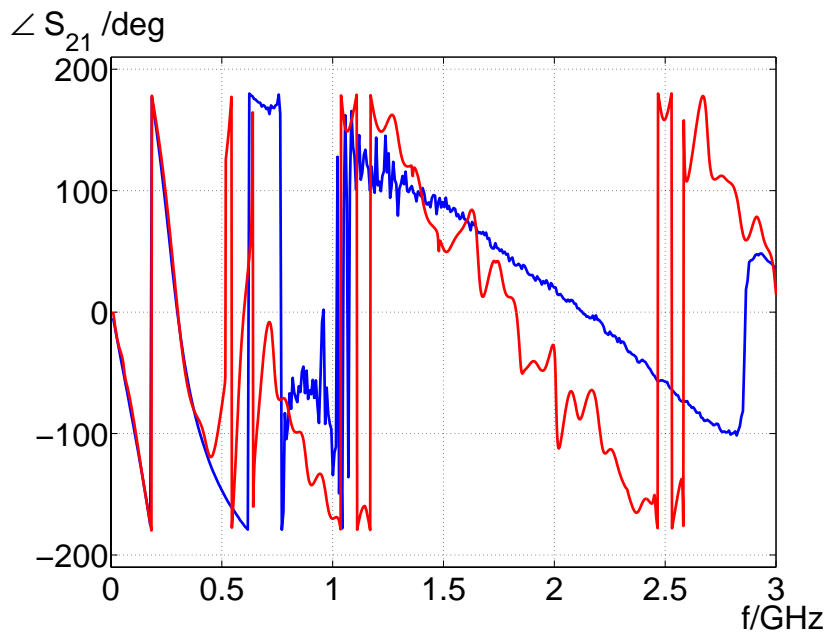

Fig. 6. Phase of $S_{21}$ of a $300 \mathrm{MHz}$ low pass filter, - network analyzer based on pulse generators, - commercial network analyzer (ZVR).

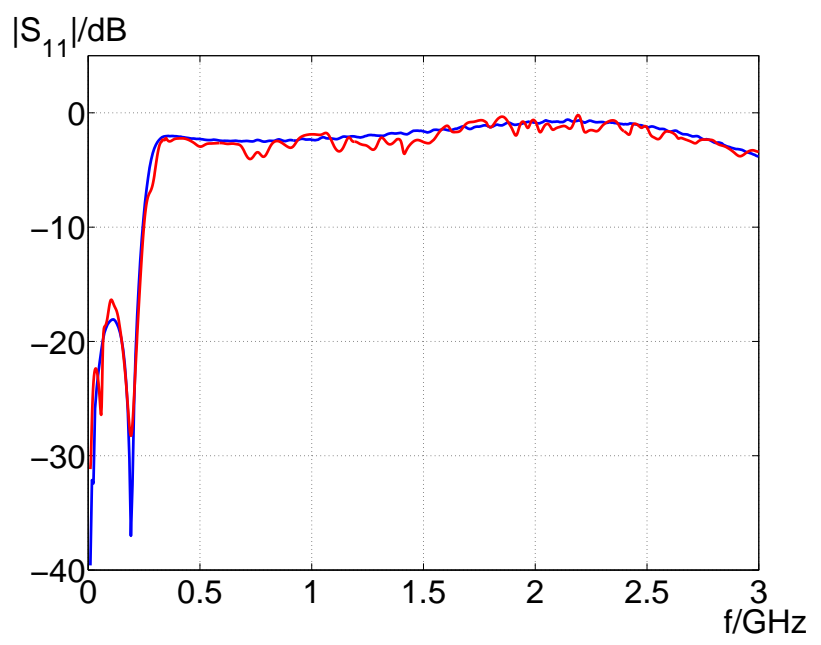

Fig. 7. Magnitude of $S_{11}$ of a $300 \mathrm{MHz}$ low pass filter, - network analyzer based on pulse generators, - commercial network analyzer (ZVR).

\section{Conclusion}

A fast network analyzer based on pulse generators has been introduced for frequencies from $10 \mathrm{MHz}$ to $3 \mathrm{GHz}$. The pulse generators used for the signal generation are realized costeffectively with bipolar transistors. This analyzer has the same structure as a conventional four channel network analyzer. Thus all known calibration techniques can be used. The analysis technique differs from the conventional one, because the data for the evaluation is recorded in the time domain and not frequency point by frequency point. For this reason the evaluation of the S-parameters is based on a Fourier transformation.

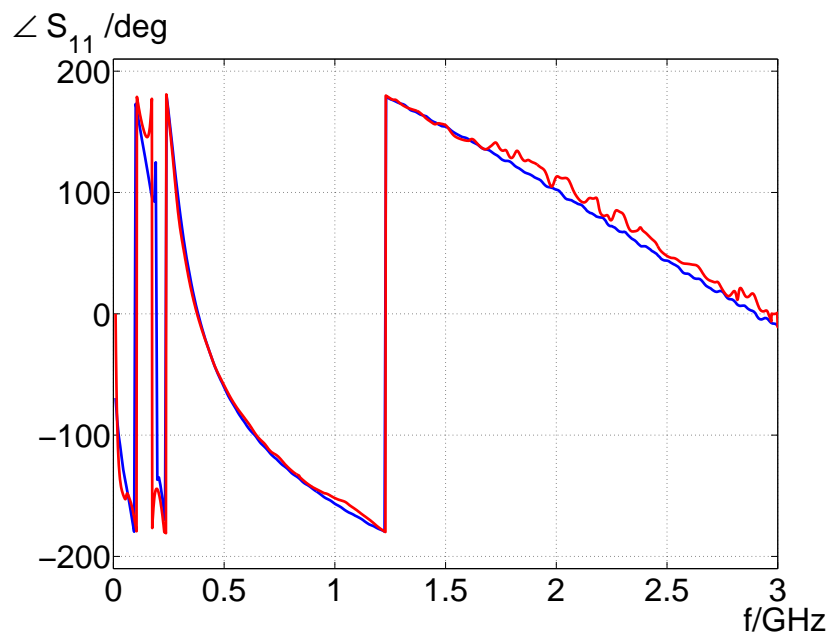

Fig. 8. Phase of $S_{11}$ of a $300 \mathrm{MHz}$ low pass filter, - network analyzer based on pulse generators, - commercial network analyzer (ZVR).

\section{References}

Gerding, M., Musch, T., and Schiek, B.: Generation of short electrical pulses based on bipolar transistors, Ad. Radio Sci., Kleinheubacher Berichte 2003, Kleinheubach, Germany, 7-12, 2003.

Schiek, B.: Grundlagen der Hochfrequenz-Messtechnik, SpringerVerlag, Germany, 1999. 